\title{
The Origin of Strain Avalanches in Sub-micron Plasticity of FCC Metals
}

\author{
Tamer Crosby, Giacomo Po, Can Erel, Nasr Ghoniem \\ Department of Mechanical $\mathcal{G}$ Aerospace Engineering, University of California at Los \\ Angeles (UCLA), 420 Westwood Plaza, Los Angeles, CA. 90095-1597, U.S.A. \\ tcrosby@ucla.edu,gpo@ucla.edu,canerel@ucla.edu,ghoniem@ucla.edu,
}

\begin{abstract}
Numerous experimental observations have recently demonstrated two aspects of sub-micron plasticity in nano pillars: (1) the statistical nature of the yield strength, and (2) the existence of dynamic temporal oscillations in the hardening regime. The present work is focused on the second aspect, where we study strain avalanches in nickel nano pillars via a series of 3-D dislocation dynamics (DD) simulations. The computer simulations reveal the existence of a key mechanism that explains the origin of oscillations in post-yield plastic flow in fcc metals. Small dipolar-loops easily form through several crossslip mechanisms, and then act as internal glide dislocation sources. When dislocations exit pillar boundaries, they leave many dipolar-loops behind. At this stage, the pillar is starved from dislocations and an increase in stress is necessary to keep up with the applied strain rate (hardening stage). At some critical stress level, dipolar-loops become effective Frank-Read sources of new glide dislocations that collectively move in a burst mode, producing strain avalanches (softening stage). The time delay between these two states, which is determined by the average dislocation velocity and pillar diameter, leads to plastic strain oscillations and intermittent dislocation avalanches, consistent with experimental observations.
\end{abstract}

Keywords: strain avalanches, dipolar-loops, dislocations, submicron plasticity, FCC metals

\section{Introduction}

Understanding the mechanisms that control plasticity at the nano and micro scales is essential in gaining insights into the physics of sub-micron plastic- 
ity, and in guiding the design of new materials with a desired combination of strength and ductility. Moreover, recent advances in small-scale mechanical testing and high-resolution material characterization offer unprecedented opportunities for validation of a physics-based approach to modeling of plastic deformation. In small scale plasticity, plastic deformation exhibits features that are not common in polycrystals at larger length scales. Specifically, the stress-strain relationship has three distinct characteristics: (1) the flow stress is higher (i.e. the smaller the diameter, the harder the material), (2) the flow stress is stochastic (i.e. different tensile experiments on the same material lead to different flow stress values), and (3) the post-yielding stress behavior displays strain oscillations accompanied with dislocation avalanches.

The influence of specimen size, at the nano and micro scales, on strength (yield point) has been investigated, both experimentally (e.g. references [112]), and via computer simulations (e.g. references[13-18]). Because the dislocation density is within a limited range $\left(\rho \approx 10^{12}-10^{14} \mathrm{~m}^{-2}\right.$ for a wellannealed material), only tens to hundreds of dislocation loops contribute to plastic strain in sub-micron plasticity. This is to be contrasted with a much larger number of glissile dislocation loops in polycrystals. Additionally, dislocations glide on many more planes in polycrystals as compared to the limited number of slip planes in typical nano/micro pillar experiments. Thus, both the yield strength and post-yield hardening are very sensitive to the way test samples are prepared, and hence to the initial distribution and density of dislocations inside the material. These two aspects (small number of glide dislocations and limited slip planes) must be responsible for the experimentally-observed strain oscillations in nano/micro pillars. Stochasticity in the flow stress is apparent in pillars with diameters less than 10 $\mu \mathrm{m}$, with an increase in the amplitude of strain oscillations for diameters less than $1 \mu \mathrm{m}$ (see Fig. 3 of reference [5]). The size effect on strength, and the stochastic nature of plastic flow were also observed in bcc metals. It was shown in reference[6] that the mechanisms of deformation in bcc metals depend on the size, similar to what is known about fcc metals. However, plastic flow is characterized by a critical temperature with a transition from continuous to jerky stress-strain behavior and a significant change in the deformation morphology between small and large samples [6]. We confine the present investigation to fcc metals, where specific mechanisms can be revealed through computer simulations.

The phenomenon of strain avalanching (or serrated yield) is not only associated with sub micron plasticity, but is well known in dynamic strain aging, 
and in plastic instabilities of polycrystals as well. The serrated yield behavior of the Portevin-Le Chatelier (PLC) effect [19, 20] is well-understood, where the mechanism that leads to strain bursts is a result of pinning of dislocations by solute atoms, and further de-pinning above a critical stress. Another example is the formation of Lüders bands, which is distinctly different from the PLC behavior. While the PLC behavior is associated with solute diffusion, and the strain bursts continue repeatedly as the specimen is strained, Lüders bands propagate only once in the specimen at essentially zero hardening rate [21]. A widely accepted underlying mechanism of Lüders bands is attributed to Cottrell and Bilby, and is initiated by de-pinning avalanches of dislocations stuck at "Cottrell interstitial atmospheres," followed by pileup formations at grain boundaries leading to subsequent hardening [22]. Lüders bands have also been observed in single crystal deformation by Cottrell and Stokes, who postulated that sessile dislocations in these crystals pin pileups, which can then be released and produce avalanches [23]. Thus, the intermittent nature of plastic flow seems to be general, but the underlying mechanisms may be dependent on the specific material and test conditions. In sub micron samples, strain avalanches have been observed in experiments (Fig. 4a, reference [7] and Fig. 3, reference [8] ), and in other examples in the literature (e.g. references $[9,13])$.

The exact fundamental processes behind the size effect on the strength of nano and micro pillars have been the subject of recent controversy. A number of mechanisms have been proposed on the basis of computer modeling and experimental observations: (1) the dislocation starvation mechanism [11], (2) the weakest link mechanism [14], (3) the source truncation or single arm source mechanism [12], and (4) the source exhaustion mechanism [15]. In general, one may divide the factors influencing strength and hardening in nano and micro pillars into two main groups: extrinsic and intrinsic effects. Extrinsic effects are the result of the boundary interaction with dislocations. This affects the average lifetime of dislocation lines inside the material, once they are created. For instance, single arm sources will be in general shorter in small diameter pillars than in larger ones, as they reach the boundary faster and hence get truncated. Intrinsic effects denote the effects of the underlying internal microstructure as dislocations interact with one another. In larger size pillars, there is a higher chance for a dislocation segment to form sessile junctions that lead to blocking of glissile dislocations, and thus result in traditional forest-hardening.

The above models, however, have mainly focused on analyzing the influ- 
ence of the sample size on the onset of plastic flow (i.e. yield point), with little attention to the characteristics of plastic flow at high strains, well beyond the yield point. Thus, a fundamental understanding of the origins of strain-avalanches and associated stress oscillations in sub-micron-size specimens is still lacking. In the present investigation, we focus our attention on the stress-strain behavior past the initial yield point in an attempt to provide a mechanistic explanation for the existence of strain avalanches and intermittent plasticity in sub-micron-size specimens. The approach here is to utilize systematic 3-D dislocation dynamics (DD) simulations that provide numerical (virtual) experimental observations of the microstructure during deformation.

The layout of the paper is as follows: in section 2 we outline briefly the method of DD with a discussion of the main constituents of the dislocation microstructure. DD simulations have shown the existence of a dynamic process of creation and destruction of dipolar-loops (acting as dislocation debris), which will be illustrated to be responsible for the hardening-softening oscillation cycles in fcc metals at the sub-micron scale. Thus, in section 4.1, we present the various mechanisms by which dipolar-loops interact with dislocation segments, followed by analysis of strain avalanches in section 4.2. Finally, the main conclusions are given in section 5 .

\section{Discrete Dislocation Dynamics simulations}

The phenomenon of post-yield stress oscillations in compressed nano pillars is simulated using the method of Discrete Dislocation Dynamics (DDD) $[24,25]$. With reference to Fig. 1, we consider a single crystal fcc Ni pillar of diameter $250 \mathrm{~nm}$ and aspect ratio 3:1. The axis of the pillar coincides with the [111] crystallographic direction. In the initial configuration, dislocation dipoles are generated inside the pillar with density $10^{12} \mathrm{~m}^{-2} \leq \rho \leq 10^{13} \mathrm{~m}^{-2}$ and with random size, glide plane, and Burgers vector. Under the influence of their mutual interaction and the applied stress, dislocations glide on their slip planes according to a mobility law, $\boldsymbol{f}=B \boldsymbol{v}$, where $\boldsymbol{B}$ and $\boldsymbol{v}$ are the mobility coefficient and the velocity vector, respectively, and $\boldsymbol{f}$ is the Peach-Koehler force. Dislocation climb is inhibited, while cross-slip is modeled as a thermally activated process with an activation enthalpy dependent on the Escaig stress [26]. Boundary conditions for the simulation domain are imposed by utilizing the superposition principle of the elastic field of dislocations in an infinite medium with an elastic boundary value solver implemented through 


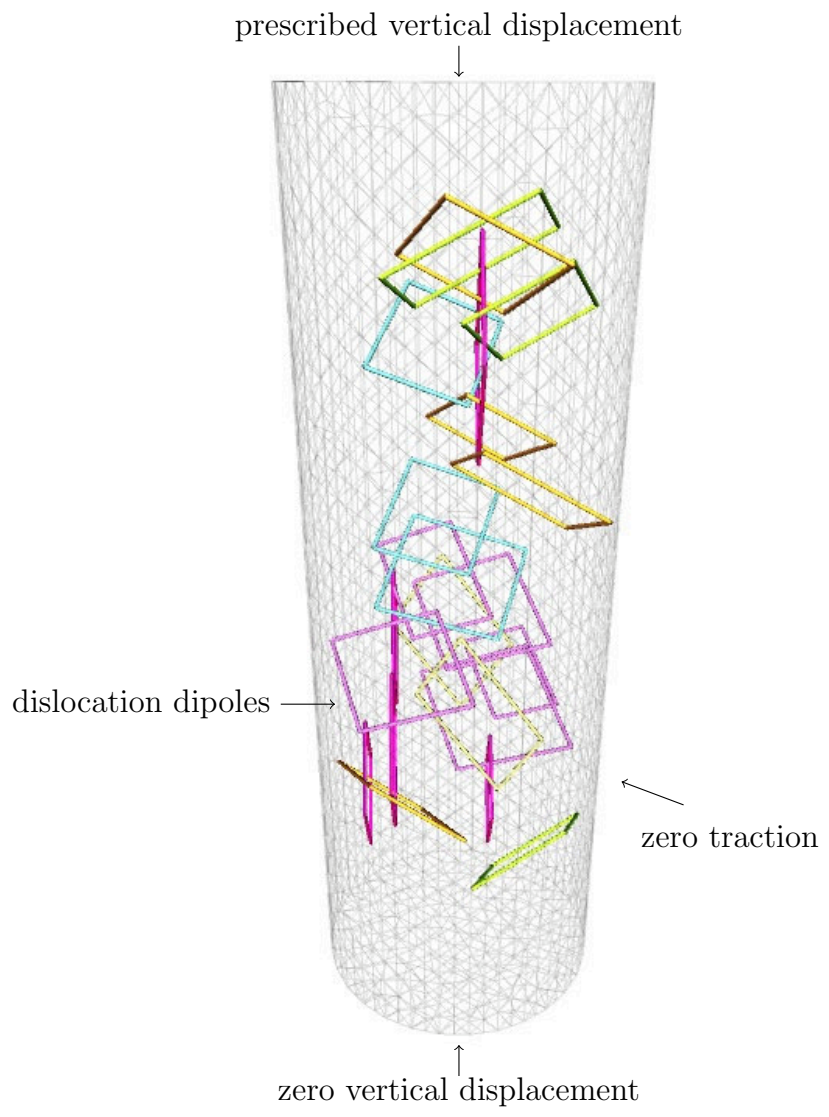

Figure 1: Nanopillar considered in our DD simulations.

the finite element method (FEM), as described in [27]. A compressive load is applied, imposing a prescribed rate of vertical displacement on the top surface of the pillar corresponding to a strain rate of $10^{4}-10^{5} \mathrm{~s}^{-1}$. The bottom surface is fixed, while the cylindrical surface is traction free.

\subsection{Initialization of the dislocation microstructure}

The choice of the initial dislocation microstructure plays an important role in DD simulations of plastic flow in in small volumes. We emphasize here that such choice involves not only the dislocation density and its spatial distribution, but also the type of dislocation sources employed. The simplest form of dislocation sources typically used in DD simulation is the Frank-Read (FR) source [e.g. references 14, 18]. While this choice is acceptable for bulk 
materials, the presence of FR sources in small domains can lead to unphysical artifacts. In fact, the open ends of $\mathrm{FR}$ sources not only violate the dislocation line continuity condition (i.e. a dislocation line cannot end inside the material [28]) but they also constitute fixed sources, which cannot be destroyed. Thus, fixed FR dislocation sources leave their imprint on the dislocation system dynamics, because they dictate both the strength and the aptitude of stress oscillations under strain control loading. In order to overcome these difficulties, the initial dislocation microstructure in the present simulations is described by dipolar-loops, with random size, orientation and location within the simulation volume (see experimental evidence in [e.g. references 29-31]). Each dipolar-loop is defined by a Burgers vector and two pairs of parallel sides that lie on two conjugate glide planes of the fcc crystal containing the Burgers vector of the loop. Because climb is inhibited, these loops are stable under their self stress. The main difference between a simple FR source and a dipolar-loop is that the latter has generally no fixed points (all sides are glissile and the corner nodes can glide on the glide cylinder), and it can thus act as a dynamic dislocation source, as discussed below. Occasionally, dipolar loops may also be generated with sessile segments, rendering them significant versatility as internal dislocation sources.

\section{Results of Computer Simulations}

DD simulations provide detailed information on the dislocation microstructure, as it evolves during a simulated micro-pillar compression test. However, the relationship between the applied stress and resulting strain component in the axial direction is useful in connecting DD simulations with experimental observations. Typical stress-strain curves obtained from DD simulations for different initial dislocation density and distributions are shown in Fig. 2. In earlier DD simulations, as well as in interpreting experimental observations, there is a tendency to ascribe a specific value for the "strength" of the micro-pillar as a function of its diameter. Thus, the concept of "smaller is stronger" attributes the strengthening effect in small-size pillars to the proximity of confining pillar walls to the interior dislocation structure, thus preventing dislocations from full interaction with other dislocations (traditional concept of hardening). The present simulations for one single size pillar show a wide range of flow strengths that vary by nearly a factor of two (see Fig. 2 ). The figure shows that the flow stress is stochastic (with a probability distribution), and that the range of the flow stress is largely determined by the 


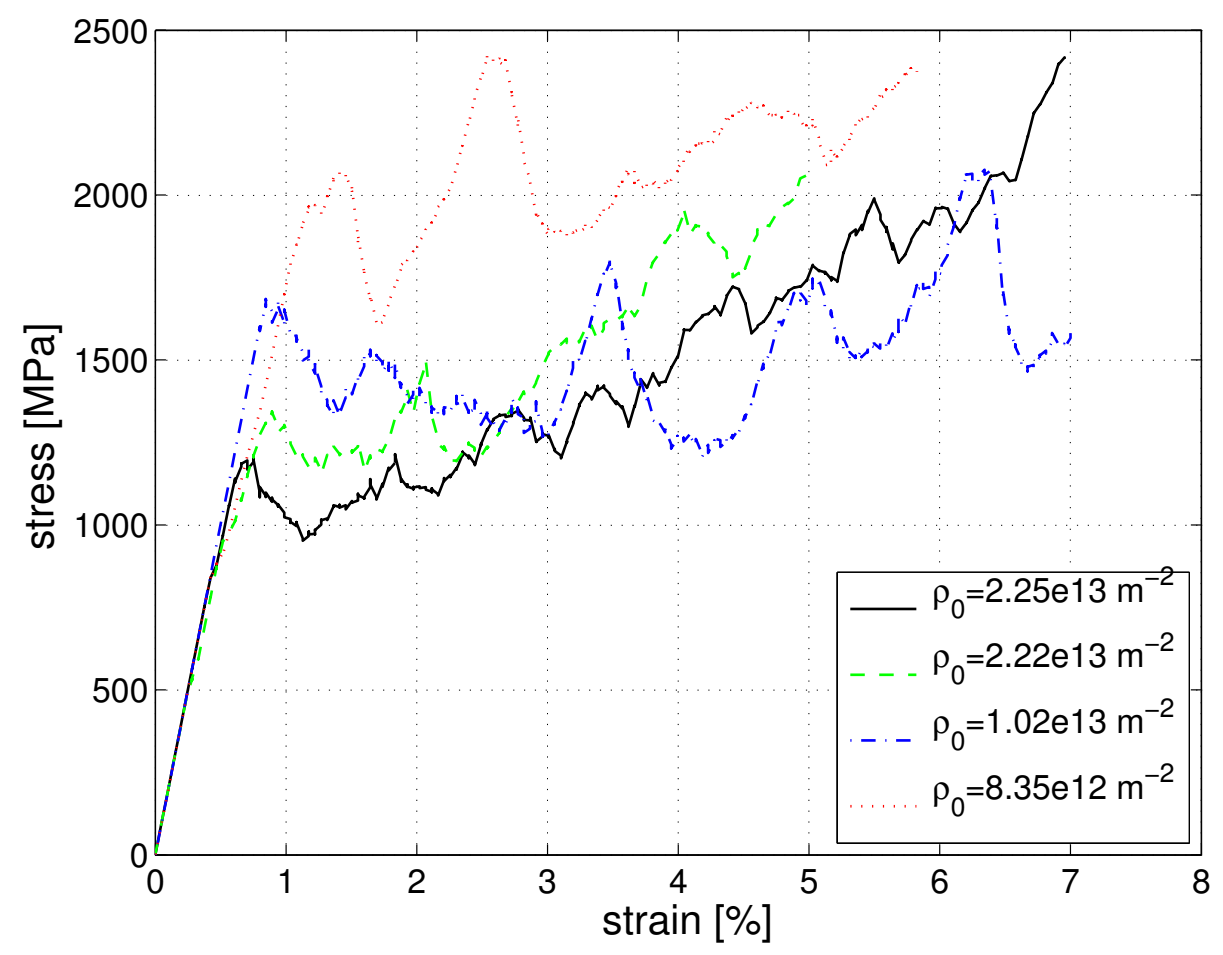

Figure 2: Stress-strain curves for a pillar under compression $(D=250 \mathrm{~nm})$ obtained from dislocation dynamics simulations. Different curves correspond to different initial dislocation densities. The flow stress is stochastic, with some dependence on the initial microstructure, followed by strain avalanches. Notice the large oscillation amplitudes for the lower initial dislocation density.

pillar size. The second general feature observed from Fig. 2 is that, beyond the yield point, there are significant oscillations of the stress, indicating the occurrence of strain avalanches within the micro pillar.

\section{Analysis of Strain Avalanches}

\subsection{Debris-Dislocation Interaction Mechanisms}

In fcc materials, there are several ways in which edge prismatic loops (dipolar-loops) can be formed. However, the main requirement for formation is the existence of a jogged dislocation. In turn, a jogged dislocation can be formed as a result of either junction formation or cross-slip. A jogged dislocation can turn into a full dipolar-loop following the classical Johnston-Gilman 
[32] or Tetelman [33] mechanisms, or from collinear reaction, as shown by Loutat and Johnson in [34]. A comprehensive study of the mechanisms of dipolar-loop formation, interactions, and their role in cyclic plasticity is detailed by the authors in reference [35].

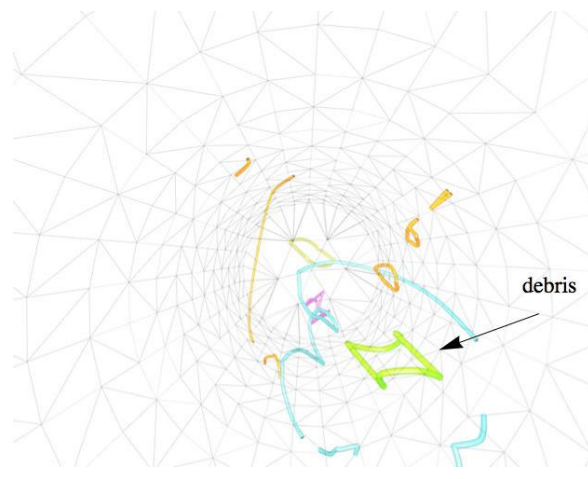

(a)

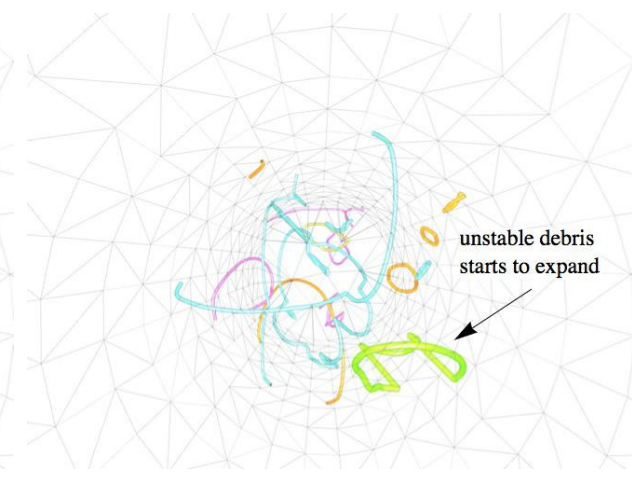

(b)

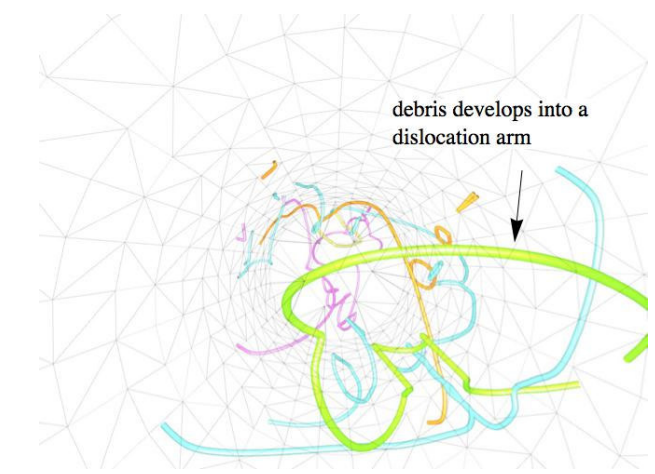

(c)

Figure 3: A series of snap shots (top-view of the pillar) showing dislocation debris turning into a source due to its instability (pointed to with an arrow). (a) The debris is unstable under the local stress field. (b) As a result, one segment starts to further expand. (c) The arm keeps expanding and starts to interact with the surrounding dislocation network and with the boundary.

Dipolar loops have been described as dislocation debris by a number of authors. Niewczas [29] used transmission electron microscopy (TEM) to observe dislocation structures in deformed copper single crystals. In addition to the regular dislocation microstructure, a large density of defects, namely dislocation edge dipoles (dipolar-loops), faulted dipoles, and stacking-fault 


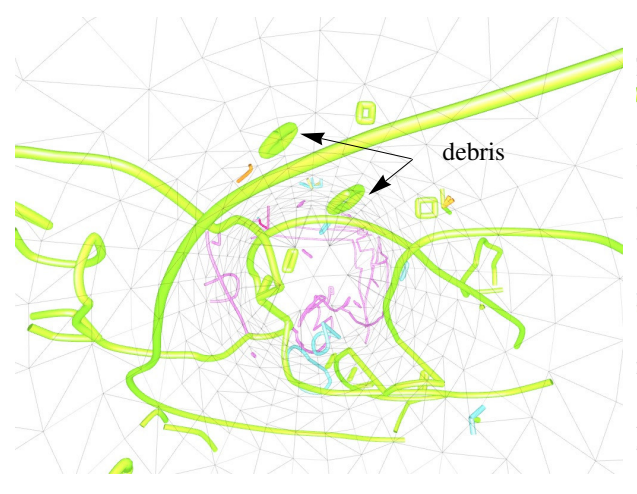

(a)

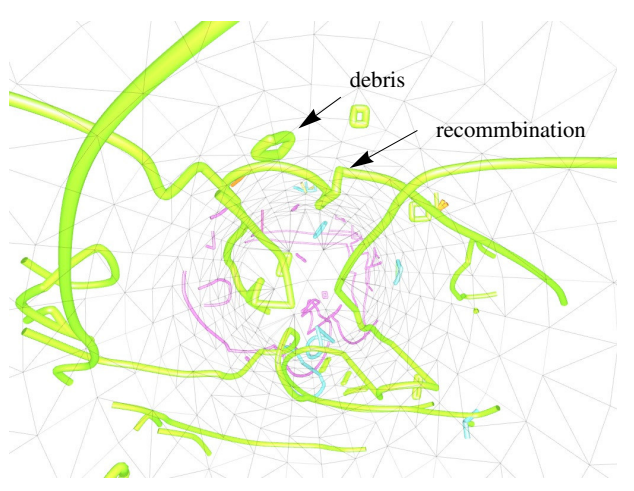

(b)

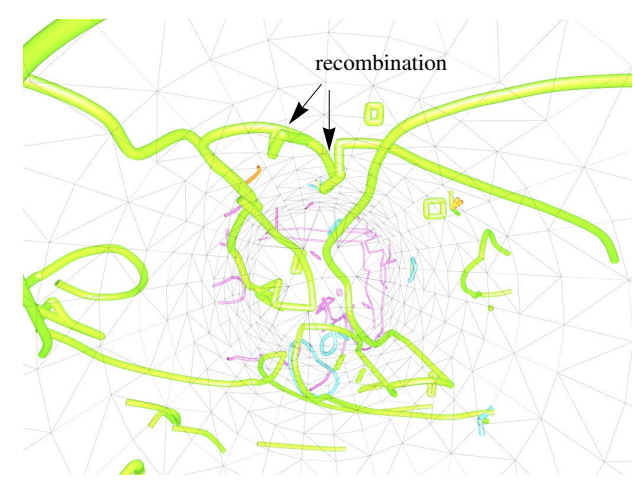

(c)

Figure 4: A series of snap shots (top-view of the pillar) showing the recombination of debris (pointed to with an arrow) with a dislocation segment. (a) Two dipolar-loops are about to interact with a dislocation segment. (b) One dipolar-loop gets annihilated with the dislocation segment. (c) The second dipolar-loop gets annihilated as well with dislocation segments. As a result, the two dipolar-loops disappear.

tetrahedra were observed, and collectively described as "dislocation debris". In his observations, Niewczas determined the size distribution of each type of these defects. In the present DD simulations, the average size of equilibrium dipolar-loops was found to be around $5 \mathrm{~nm}$. This matches the experimental debris sizes reported by Niewczas for the same range of applied stress. The DD simulations show that the debris, once formed, reacts with the dislocation network according to four different possibilities. The first is a debris "burst mode", Fig. 3, in which highly-stressed dipolar loops that are not in equilibrium, become unstable and act as dislocation sources by bursting into 


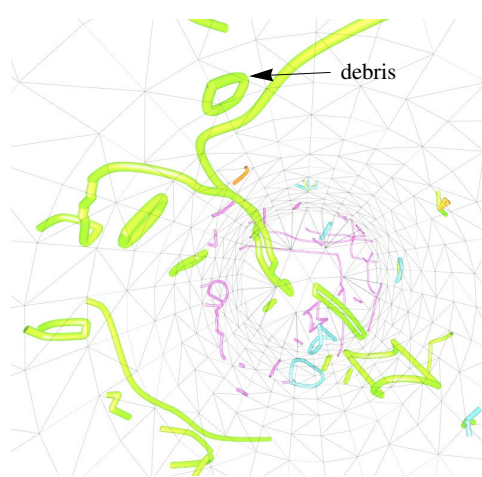

(a)

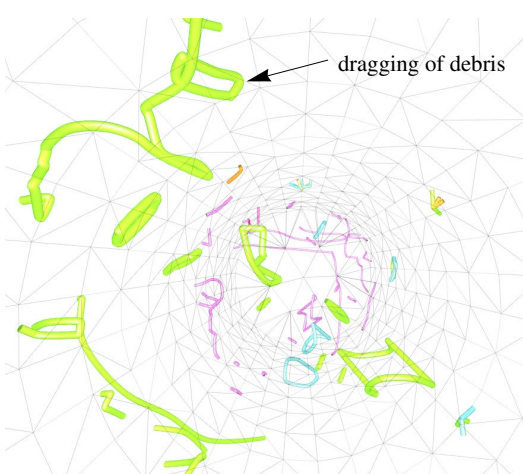

(b)

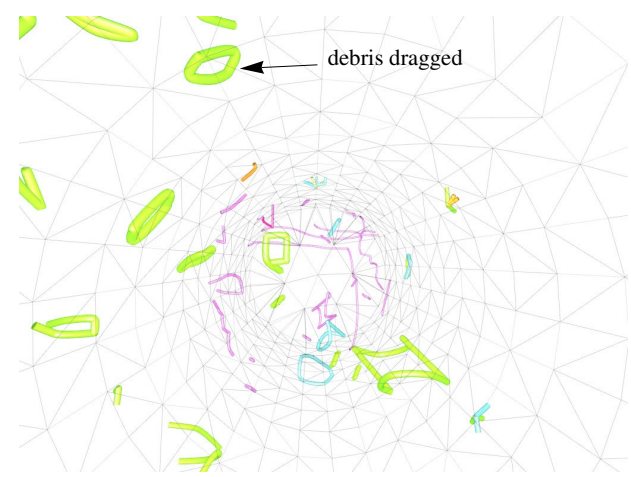

(c)

Figure 5: A series of snap shots (top-view of the pillar) showing the dragging of debris (pointed to with an arrow) (a) A dipolar-loop is about to interact with a passing dislocation segment. (b) The dislocation segment exerts forces on the dipolar-loop. (c) As a result, the dipolar-loop gets dragged to a new position.

full dislocation segments. The second scenario is a debris "recombination mode", Fig. 4, in which dislocation lines interact with dipolar loop debris by annihilating one of the segments and incorporating it as a part of the dislocation network. In addition, some of the dipolar-loops might, when they interact with one another, form single and double arm sources. The arm sources may eventually leave the material by a multiple series of cross-slips, which is referred to as wavy-slip as opposed to planar slip. The third scenario is a debris "drag mode", Fig. 5, in which the stress field from a passing dislocation line drags a dipolar loop debris for a short distance. The fourth scenario is when dipolar loop debris gets stuck and just stay in the material, 
"trapping mode", influencing the stress-field of the dislocation network, but without any direct topological interaction with another dislocation line. As a result, some of the dipolar-loops or debris will act as obstacles. However, these obstacles might only be present temporarily before being destroyed, or they may occasionally contain sessile segments providing permanent obstacles to dislocation motion.

\subsection{Origins of Strain Avalanches}

The phenomenon of strain avalanches have been explored by multiple authors using various experimental and computational techniques. In bulk materials, for instance, acoustic emissions (AE) analysis has been widely used to investigate strain bursts and avalanches in metals. For example, in reference [36] the Portevin-Le Chatelier effect in Al-Mg alloy was studied, and it was shown that the statistics of catastrophic slip follows a power law distribution. In reference[37], a scale-free power law distribution of strain amplitude was observed during single slip of copper single crystals. Acoustic emissions have been used as well in ice crystals [38-40]. However, ice crystals are highly anisotropic and cannot directly be compared to isotropic metal crystals. While dislocation avalanches lead to sudden increase in the plastic strain in micro pillars (known as strain bursts), this behavior is not common in polycrystals, where the stress-strain curves appear to be very smooth and without oscillations. In reference [41], DD simulations were used to analyze the dependence of the avalanche amplitude on the system size. However, the ends of F-R sources in such simulations appear to be all pinned (see Fig. (2) of reference [41]). To date, AE has not been used to determine the dislocation avalanche statistics in nano and micro pillars. The frequency range of AE signals can be significantly higher than bulk specimens or large single crystals, adding to the challenge in the experimental application of AE to nano and micro specimens. More recently, Ispanovity and coworkers [42] have addressed the question of avalanche statistics, using 2-D and 3-D DD simulations. Their analysis of the dislocation velocity distribution function indicates that it possesses universal cubic decay at smaller velocities, and a shoulder as a result of dislocation avalanches. These conclusions seem to be based on 2-D statistics, as obtaining adequate statistics for 3-D simulations is computationally challenging at the present time. In our effort here, we focus on one particular aspect of the physics of dislocation avalanches, and that is their origin and subsequent behavior in small size cylindrical micropillars. A series of DD simulations were performed according to the setup outlined in 
section 2 for a micropillar that is $250 \mathrm{~nm}$ in diameter and $750 \mathrm{~nm}$ tall. To explore the dependence of strain avalanche amplitude on the initial dislocation microstructure, the dislocation density was varied in the range of $10^{12}-10^{13}$ $\mathrm{m}^{-2}$.

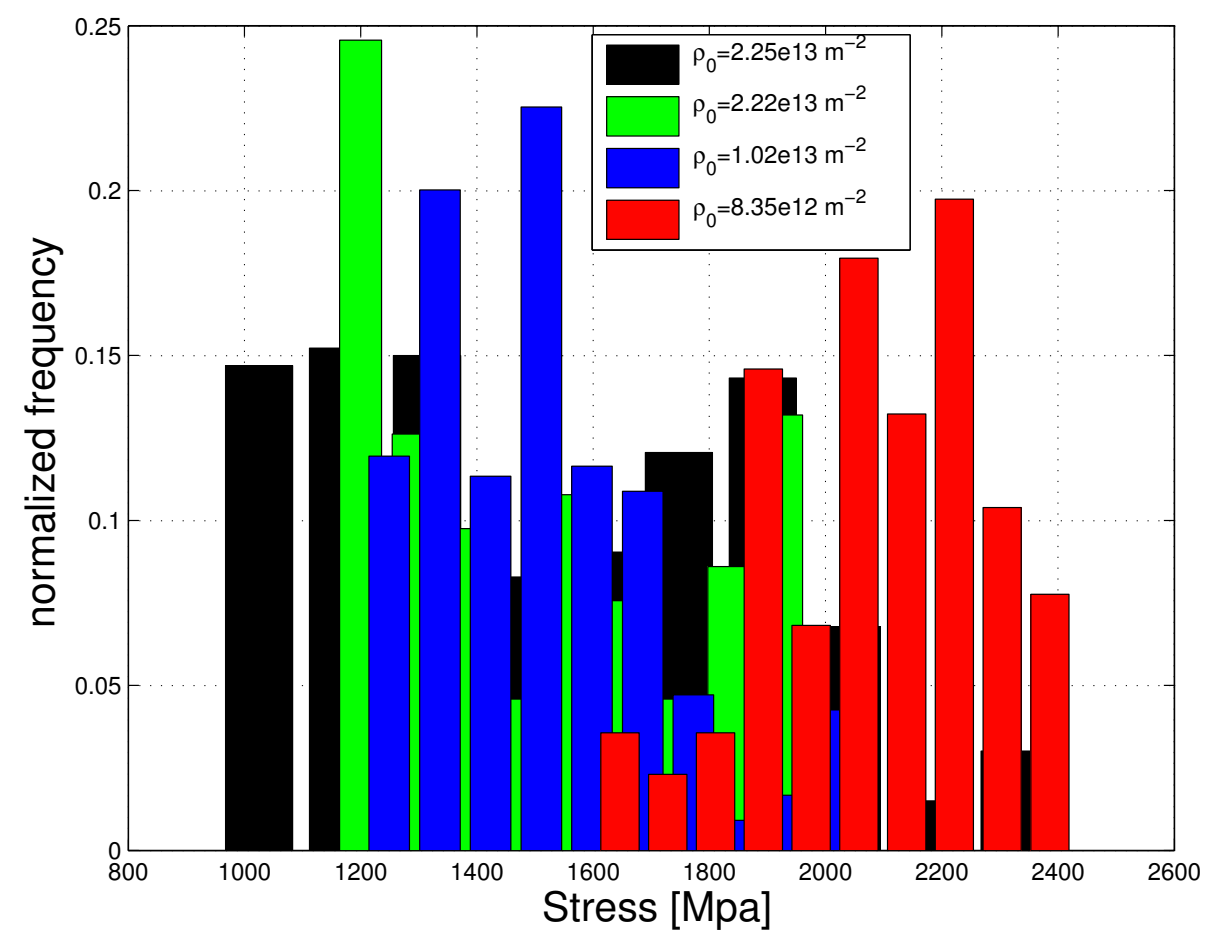

Figure 6: Analysis of stress fluctuations- past the initial yield point- for the original curves shown in Fig. 2. Histograms of stress amplitudes at different initial dislocation densities are shown.

To understand the mechanisms of strain avalanche formation, we utilize DD simulations to probe into the dynamic evolution of the dislocation network. Because of the small volume in nano/micro pillars, variations in the initial dislocation density would be expected to influence the dynamics of strain oscillations during plastic flow. Thus, the initial density can be a simple experimental parameter, which may reveal how strain bursts occur at the nano/micro scale. To shed some light on the dynamics of strain avalanches, we investigate the relationship between the initial dislocation density and the distribution and magnitude of strain bursts. Stress-strain curves, shown in 
Fig. 2, were analyzed starting form their respective initial yield point (i.e. only the plastic behavior). While it is clear from the figures that the magnitude of stress (strain) avalanches increases with smaller initial dislocation density, this dependency can be revealed by performing statistical analysis on the stress (strain) oscillation magnitude, or through stress (strain) signal processing in the frequency domain. The dependence of the stress amplitude statistics on the initial density is shown in Figure 6. Histograms for the distribution functions of stress amplitudes are shown in the figure, where the frequency of stress amplitudes is plotted against the stress for various initial dislocation density. Note that the largest effects are associated with the smallest initial dislocation density $\left(\rho_{i}=8.35 \times 10^{12} \mathrm{~m}^{-2}\right)$. One can deduce from the results that there is a clear shift in stress amplitude distributions to lower values, as the initial dislocation density increases. In other words, it is observed that the higher the initial dislocation density, the larger the damping effect on stress (strain) oscillations. The results of this statistical analysis suggest that there is an inverse relationship between the initial dislocation density and the magnitude of the stress oscillation (strain bursts). This is intuitively expected, since dislocations will have more space to expand in specimens with lower dislocation density, leading to higher magnitudes of strain bursts.

In light of the four main interaction mechanisms between dipolar loops and dislocations (burst, recombination, drag, and trapping), we can further understand the role dipolar-loops play in creating strain avalanches through analysis of the dislocation microstructure evolution. In Fig. 7(a), the microstructure is composed of a random distribution of dislocation network. When the stress reaches a value that is large enough to bow out the weakestlink (largest unpinned dislocation segment with the most favorable shear stress), dipolar-loops start to grow and the stress reaches its first yield point (Fig. 7(b)). Eventually, different dislocation segments interact with one another, as well as with the boundary, leaving behind them a trail of debris (dipolar loops of various sizes). As a result, the micropillar is left in a nearlystarved condition, with only debris left within the material ( see Fig. 7(c)). The applied stress starts to rise again in order to keep up with a fixed applied strain rate, since most of the debris does not readily deform. Finally, when the stress reaches a critical value, the debris becomes the source of fresh dislocations that readily expand to keep up with the applied strain rate (Fig. $7(d))$. When fresh dislocation segments continue to expand at a fast rate, and since the applied strain rate is fixed, the stress drops as a consequence, 


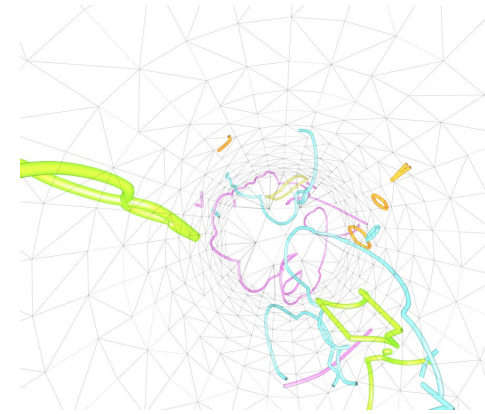

(a) nearly-starved $(\epsilon=0.08 \%)$

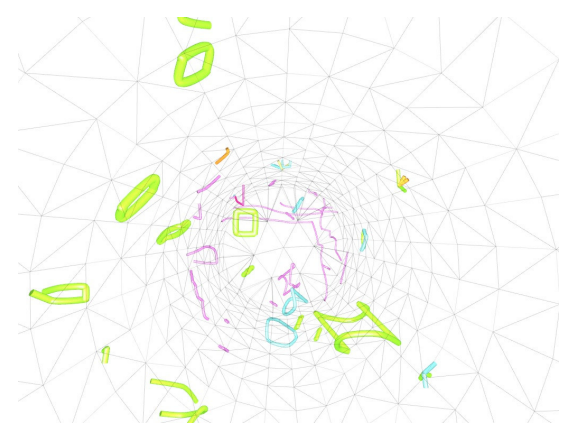

(c) nearly-starved $(\epsilon=1.22 \%)$

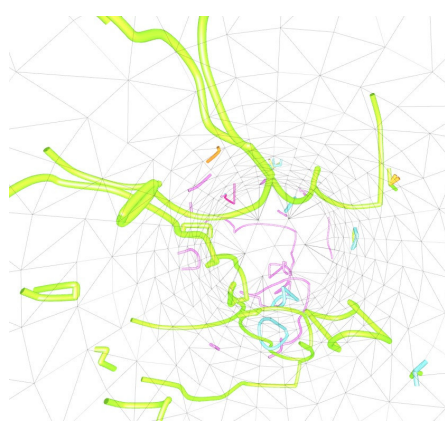

(b) full $(\epsilon=0.9 \%)$

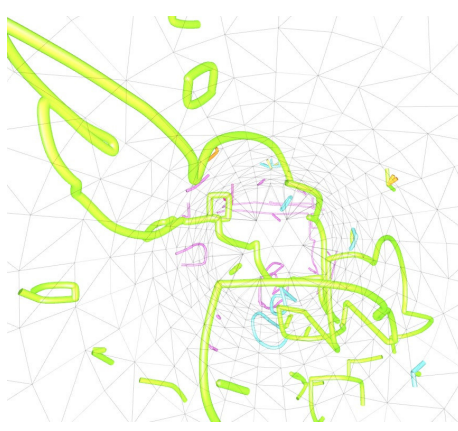

(d) full $(\epsilon=1.95 \%)$

Figure 7: A series of snap shots (top-view of the pillar) showing the evolution of microstructure inside the material. The corresponding points on the stress-strain curve are shown in Fig. 8(a) (see explanation in the body of the text).

and another wave of interactions takes place between different dislocation populations. The drop in stress is physically attributed to the fact that the tensile testing machine does not keep pace with the instantaneous vertical displacement of the specimen.

As a result of dislocation nucleation-motion cycles, the micropillar switches between two dominant states: a "dislocation-starved" state (e.g. Fig. 7(c)) and "dislocation-rich"state (e.g. Fig. 7(d)). The former is characterized by relatively small amplitude dislocation motion and correspondingly small accumulated plastic stain. This results in the build-up of applied stress to compensate for the smaller levels of plastic strain increments, and hence such larger stress values can activate new dislocation sources. On the contrary, the "dislocation-rich" state is characterized by relatively higher amplitudes of dislocation motion resulting in larger accumulated plastic strain, eventu- 


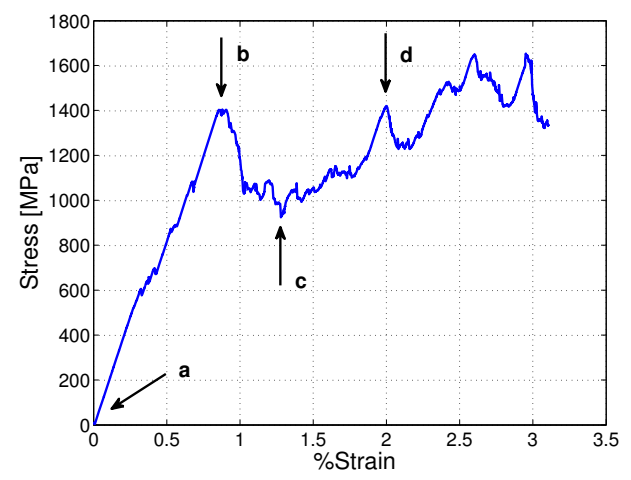

(a) stress-strain

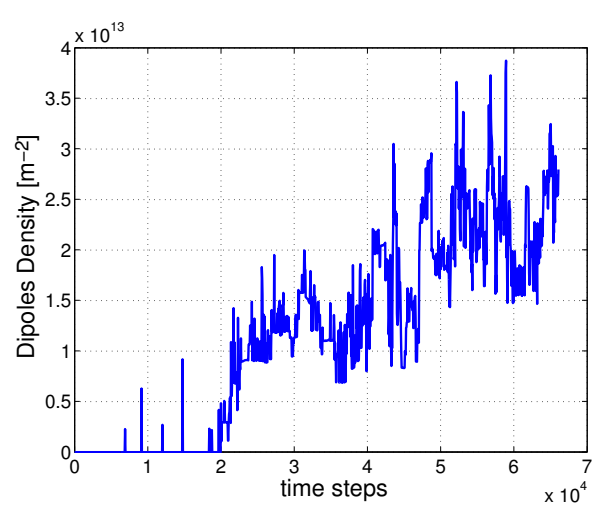

(c) $\rho_{\text {dipolar-loops }}$

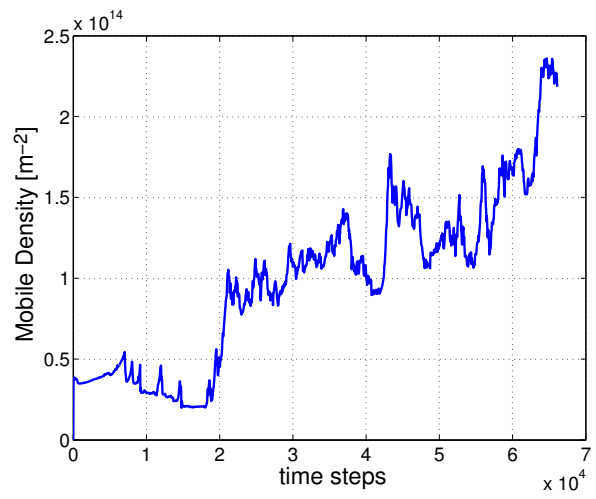

(b) $\rho_{\text {glissile }}$

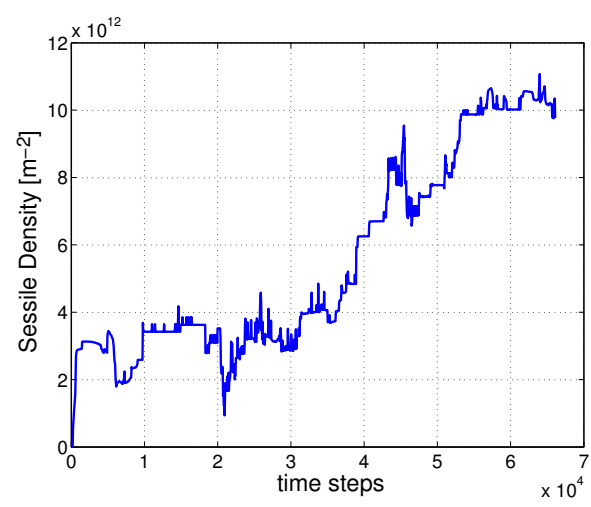

(d) $\rho_{\text {sessile }}$

Figure 8: DD simulations results (a) Stress-strain curve. (b)-(d) Time evolution of glissile, dipolar-loop, and sessile densities, respectively.

ally initiating a strain avalanche. Since dipolar-loops are continually formed and destroyed, these two states keep repeating in a dynamic but not identical manner, resulting in the formation of strain avalanches with different amplitudes and distributions. The reason that the repeating cycles are not identical is the spatial dependence of dipolar loops as dislocation sources. Dipolar loops can be dragged from one location to another inside the pillar, can have a size distribution, can have various orientations, and thus provide all the elements for a truly "stochastic" source of dislocations. An important point to make here is that if simple F-R sources (with traditionally fixed ends) were used to model dislocation sources, the complex hardening-softening be- 
havior of the stress-strain curve cannot be captured. The present model is also consistent with numerous experimental observations on micropillar compression that exhibit the stochastic behavior observed in our simulations. If artificially pinned F-R sources were used to seed the microstructure, sources will keep repeating themselves resulting in a serrated stress-strain curve, with nearly constant amplitude and periodicity. This observation is all the more important in sub-micron pillars, since the number of dislocations in the pillar is relatively small, and fluctuations in strain amplitudes are not totally damped via dislocation-dislocation interactions. However, in larger samples, and when there is larger populations of dislocations, dislocation-dislocation interaction events dominate, making the choice of initially fixed F-R sources as a starting point not very critical.

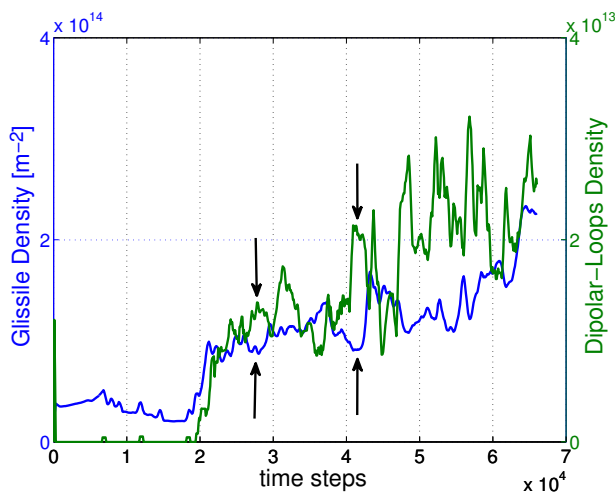

(a) $\rho_{\text {glissile }}, \rho_{\text {dipolar-loops }}$ vs. time

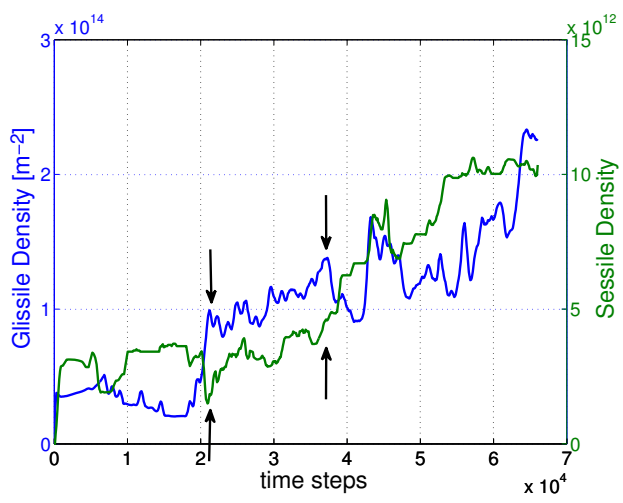

(b) $\rho_{\text {glissile }}, \rho_{\text {sessile }}$ vs. time

Figure 9: DD simulations results (a) Time evolution of glissile and dipolar-loop densities. (b) Time evolution of glissile and sessile densities.

In order to confirm the key role that dipolar-loops play in causing strain avalanches, we divide the evolving dislocation network into three families: glissile, sessile, and dipolar-loops, such that:

$$
\rho_{\text {total }}=\underbrace{\rho_{\text {glissile }}}_{\text {mobile }}+\underbrace{\rho_{\text {sessile }}}_{\text {immobile }}+\underbrace{\rho_{\text {dipolar-loops }}}_{\text {mobile } / \text { immobile }}
$$

where $\rho_{i}\left(\mathrm{~m}^{-2}\right)$ is the dislocation density for family " $i$ ". Glissile segments are completely mobile, sessile segments have zero mobility, and dipolar-loops are either mobile or immobile, depending on how they interact with the 
dislocation network. With the knowledge of nodal connectivity, slip planes, and Burgers vector of each segment in the network, we can exactly calculate the density of dislocations in each one of the three families.

The stress-strain curve and dislocation densities of the three dislocation families are plotted for a representative case in Fig. 8. The stress-strain curve is shown in Fig. 8(a), while Figs. 8(b), 8(c), and 8(d) display the evolution of the glissile, dipolar-loops, and sessile densities, respectively. It is clear that the three densities exhibit a multistage oscillating behavior, where they generally increase with strain, but within each stage they display small amplitude oscillations. Strain oscillations (with occasional bursts) are attributed to the influence of system size (boundary effects), which is very prominent in small scale plasticity. This behavior is nearly absent in polycrystals, or in large-size systems. The overall buildup in the three densities is a result of dislocation immobilization by formation of sessile segments (cf. Fig. 8(d)), formation of dislocation pileups, and the preponderance of dipolar loops acting as further obstacles to dislocation motion.

Fig. 8 shows that the intermittent behavior of the stress-strain profile is closely coupled with the stochastic oscillations in the three dislocation populations. To further investigate the role played by each one of the three dislocation families, we plot the evolution of the dipolar-loop and glissile densities superimposed on one another in Fig. 9(a). A similar plot is shown for the sessile and glissile densities in Fig. 9(b). The plots show that the glissile and dipolar-loop densities have, overall, opposite trends with respect to their maxima and minima (the populations are out of phase). This explains the switch between the two perviously discussed states of the material (the "dislocation-starved," which corresponds to a peak in the dipolar-loop density and a valley in the glissile density, and vice-versa for the "dislocationrich" state). As a result, one can infer that the dynamic creation and destruction of dipolar-loops is the key mechanism that determines the onset of strain avalanches in sub-micron plasticity. This conclusion cannot be reached with 2-D DD simulations alone, as they lack information on the 3-D topology that is needed to create and destroy dipolar loops in a random fashion. It is also necessary to solve the coupled boundary value problem with DD simulations so as to properly account for boundary effects on the confinement and absorption of dislocation segments at the pillar boundaries. 


\section{Conclusions}

We investigated here the underlying physics of strain avalanches and intermittent plasticity in sub-micron pillars tested in compression. At this length scale, the mechanical behavior is distinctly different from that of bulk materials. The dynamics, mutual and geometrical interactions of dislocation segments have a very strong influence on the overall behavior of the stressstrain relation, and hence on the micromechanics of the material. A salient aspect of the present simulations is that there are no artificially pinned dislocation sources inside the volume. Therefore, dislocation sources are created and destroyed in a continuous fashion, breaking any attempt of uniform periodicity in the dynamics of dislocations. This intriguing aspect of dynamics is rooted in the space-time stochasticity of dislocation sources themselves (here they are the dipolar loops). In larger volumes, on the contrary, where the number of dislocations is relatively larger, the shape of the stress-strain curve and the properties of the macroscopic mechanical properties of the material are dictated by the aggregate or collective behavior of dislocations.

The jerky behavior of the stress is intrinsic and a property of small scale systems, and not the result of some external fluctuations or randomness. We suggest, based on the simulation results, that this is attributed to the nonlinear dynamics of formation and destruction of dislocation debris (dipolar loops). The debris-mechanism for intermittent plasticity appears to be fundamental to the understanding of plastic flow in confined, sub-micron plasticity. An analysis for the evolution of dislocation densities that correspond to different dislocation families, confirms the role dipolar loops play in dictating the onset of strain avalanches. The analysis suggests that the continuous shift in the peaks and valleys of the glissile and dipolar-loops densities correspond to the continuous space-time shifts of sources inside the material.

Our conclusions on the connection between the formation of dipolar-loop debris and strain avalanches confirms the simplified analysis carried by Neumann [43] on slip instabilities and strain-bursts (avalanches). In his work on fcc single crystals under cyclic deformation, he concluded that decomposition of dipolar-loops happens more often than trapping of approaching dislocations. This results in a significant dipolar-loop instability under an applied stress which causes pronounced strain-bursts as was observed experimentally [44]. In addition, our classification for the possible interaction scenarios between dipolar-loops and dislocation segments (i.e. burst, drag, 
recombination, and trapping) is consistent with Neumann's observations of stain bursts under fatigue conditions [43]. This shows that dipolar loop debris play a similar role in controlling strain-avalanches in sub-micron plasticity as they do during cyclic loading of bulk materials.

\section{Acknowledgments}

This work is supported by the Air Force Office of Scientific Research (AFOSR), award number FA9550-11-1-0282 with UCLA, and with partial support from the Department of Energy (DOE), Office of Fusion Energy Sciences through grant number DE-FG02-03ER54708:014.

\section{References}

\section{References}

[1] M. D. Uchic, D. M. Dimiduk, J. N. Florando, W. D. Nix, Sample dimensions influence strength and crystal plasticity, Science 305 (5686) (2004) 986-989.

[2] D. M. Dimiduk, C. Woodward, R. LeSar, M. D. Uchic, Scale-free intermittent flow in crystal plasticity, Science 312 (5777) (2006) 1188-1190.

[3] A. T. Jennings, M. J. Burek, J. R. Greer, Microstructure versus size: mechanical properties of electroplated single crystalline $\mathrm{Cu}$ nanopillars, Physical review letters 104 (13) (2010) 135503.

[4] J. R. Greer, J.-Y. Kim, M. J. Burek, The in-situ mechanical testing of nanoscale single-crystalline nanopillars, JOM 61 (12) (2009) 19-25.

[5] M. D. Uchic, P. A. Shade, D. M. Dimiduk, Plasticity of micrometer-scale single crystals in compression, Annual Review of Materials Research 39 (2009) 361-386.

[6] A. Schneider, D. Kaufmann, B. Clark, C. Frick, P. Gruber, R. Mönig, O. Kraft, E. Arzt, Correlation between critical temperature and strength of small-scale bcc pillars, Physical review letters 103 (10) (2009) 105501.

[7] S.-W. Lee, S. M. Han, W. D. Nix, Uniaxial compression of fcc Au nanopillars on an $\mathrm{MgO}$ substrate: The effects of prestraining and annealing, Acta Materialia 57 (15) (2009) 4404-4415. 
[8] A. T. Jennings, J. Li, J. R. Greer, Emergence of strain-rate sensitivity in $\mathrm{Cu}$ nanopillars: Transition from dislocation multiplication to dislocation nucleation, Acta Materialia 59 (14) (2011) 5627-5637.

[9] W. D. Nix, J. R. Greer, G. Feng, E. T. Lilleodden, Deformation at the nanometer and micrometer length scales: Effects of strain gradients and dislocation starvation, Thin Solid Films 515 (6) (2007) 3152-3157.

[10] A. Kunz, S. Pathak, J. R. Greer, Size effects in Al nanopillars: Single crystalline vs. bicrystalline, Acta Materialia 59 (11) (2011) 4416-4424.

[11] J. R. Greer, W. D. Nix, Nanoscale gold pillars strengthened through dislocation starvation, Physical Review B 73 (24) (2006) 245410.

[12] D. Kiener, A. Minor, Source truncation and exhaustion: insights from quantitative in situ TEM tensile testing, Nano letters 11 (9) (2011) 3816-3820.

[13] J. R. Greer, C. R. Weinberger, W. Cai, Comparing the strength of fcc and bcc sub-micrometer pillars: Compression experiments and dislocation dynamics simulations, Materials Science and Engineering: A 493 (1) (2008) 21-25.

[14] J. A. El-Awady, M. Wen, N. M. Ghoniem, The role of the weakest-link mechanism in controlling the plasticity of micropillars, Journal of the Mechanics and Physics of Solids 57 (1) (2009) 32-50.

[15] C. Zhou, I. J. Beyerlein, R. LeSar, Plastic deformation mechanisms of fcc single crystals at small scales, Acta Materialia 59 (20) (2011) 76737682 .

[16] C. Zhou, S. B. Biner, R. LeSar, Discrete dislocation dynamics simulations of plasticity at small scales, Acta Materialia 58 (5) (2010) 15651577.

[17] S.-W. Lee, W. D. Nix, Geometrical analysis of 3D dislocation dynamics simulations of FCC micro-pillar plasticity, Materials Science and Engineering: A 527 (7) (2010) 1903-1910.

[18] D. Weygand, M. Poignant, P. Gumbsch, O. Kraft, Three-dimensional dislocation dynamics simulation of the influence of sample size on the 
stress-strain behavior of fcc single-crystalline pillars, Materials Science and Engineering: A 483 (2008) 188-190.

[19] A. Van den Beukel, Theory of the effect of dynamic strain aging on mechanical properties, Physica status solidi (a) 30 (1) (1975) 197-206.

[20] S. D. Mesarovic, Dynamic strain aging and plastic instabilities, Journal of the Mechanics and Physics of Solids 43 (5) (1995) 671-700.

[21] A. Wijler, J. Schade van Westrum, On the difference between Lüders bands and portevinLe chatelier bands, Scripta Metallurgica 5 (10) (1971) 821-824.

[22] S. Kyriakides, J. Miller, On the propagation of Luders bands in steel strips, Journal of Applied Mechanics 67 (4) (2000) 645-654.

[23] A. Cottrell, R. Stokes, Effects of temperature on the plastic properties of aluminium crystals, Proceedings of the Royal Society of London. Series A. Mathematical and Physical Sciences 233 (1192) (1955) 17-34.

[24] N. Ghoniem, M, S.-H. Tong, L. Sun, Parametric dislocation dynamics: a thermodynamics-based approach to investigations of mesoscopic plastic deformation, Physical Review B 61 (2) (2000) 913.

[25] G. Po, N. M. Ghoniem, A variational formulation of constrained dislocation dynamics coupled with heat and vacancy diffusion, J Mech Phys Solids 66 (2014) 103-116.

[26] B. R. Ramírez, N. Ghoniem, G. Po, Ab initio continuum model for the influence of local stress on cross-slip of screw dislocations in fcc metals, Physical Review B 86 (9) (2012) 094115.

[27] G. Po, M. Mohamed, T. Crosby, C. Erel, A. El-Azab, N. M. Ghoniem, Recent progress in Discrete Dislocation Dynamics and its applications to micro plasticity, Jom-J Min Met Mat S .

[28] J. P. Hirth, J. Lothe, Theory of dislocations .

[29] M. Niewczas, Transmission electron microscopy observations of debris structure in deformed copper single crystals, Philosophical Magazine A 82 (2) (2002) 393-414. 
[30] P. Veyssière, F. Grégori, Properties of $\{110](111\}$ slip in Al-rich $\gamma$-TiAl deformed at room temperature II. The formation of strings of prismatic loops, Philosophical Magazine A 82 (3) (2002) 567-577.

[31] C. Carter, P. Hirsch, The formation and glide of jogs in low stackingfault energy face-centred cubic materials, Philosophical Magazine 35 (6) (1977) 1509-1522.

[32] W. Johnston, J. Gilman, Dislocation multiplication in lithium fluoride crystals, Journal of Applied Physics 31 (4) (1960) 632-643.

[33] A. Tetelman, Dislocation dipole formation in deformed crystals, Acta metallurgica 10 (9) (1962) 813-820.

[34] N. Loutat, C. Johnson, The behaviour of jogs in dislocations, Philosophical Magazine 7 (84) (1962) 2051-2057.

[35] C. Erel, T. Crosby, G. Po, N. Ghoniem, Dipolar Loop Formation and Interaction Mechanisms in fcc Metals, in preparation .

[36] M. Lebyodkin, Y. Brechet, Y. Estrin, L. Kubin, Statistics of the catastrophic slip events in the Portevin-Le Châtelier effect, Physical review letters 74 (23) (1995) 4758.

[37] J. Weiss, T. Richeton, F. Louchet, F. Chmelik, P. Dobron, D. Entemeyer, M. Lebyodkin, T. Lebedkina, C. Fressengeas, R. J. McDonald, Evidence for universal intermittent crystal plasticity from acoustic emission and high-resolution extensometry experiments, Physical Review B 76 (22) (2007) 224110.

[38] J. Weiss, D. Marsan, Three-dimensional mapping of dislocation avalanches: clustering and space/time coupling, Science 299 (5603) (2003) 89-92.

[39] T. Richeton, J. Weiss, F. Louchet, Breakdown of avalanche critical behaviour in polycrystalline plasticity, Nature Materials 4 (6) (2005) 465469 .

[40] T. Richeton, J. Weiss, F. Louchet, Dislocation avalanches: Role of temperature, grain size and strain hardening, Acta materialia 53 (16) (2005) 4463-4471. 
[41] F. F. Csikor, C. Motz, D. Weygand, M. Zaiser, S. Zapperi, Dislocation avalanches, strain bursts, and the problem of plastic forming at the micrometer scale, Science 318 (5848) (2007) 251-254.

[42] P. D. Ispánovity, I. Groma, G. Györgyi, F. F. Csikor, D. Weygand, Submicron plasticity: yield stress, dislocation avalanches, and velocity distribution, Physical review letters 105 (8) (2010) 085503.

[43] P. Neumann, The interactions between dislocations and dislocation dipoles, Acta Metallurgica 19 (11) (1971) 1233-1241.

[44] R. Neumann, P. Neumann, Strain bursts and coarse slip during cyclic deformation of FCC and HCP single crystals, Scripta Metallurgica 4 (8) (1970) 645-649. 